\title{
Front Matter: Volume 6892
}

, "Front Matter: Volume 6892," Proc. SPIE 6892, Ultrafast Phenomena in Semiconductors and Nanostructure Materials XII, 689201 (11 March 2008); doi: $10.1117 / 12.791598$

SPIE Event: Integrated Optoelectronic Devices 2008, 2008, San Jose, California, SPIE. United States 


\title{
PROCEEDINGS OF SPIE
}

\section{Ultrafast Phenomena in Semiconductors and Nanostructure Materials XII}

\author{
Jin-Joo Song \\ Kong-Thon Tsen \\ Markus Betz \\ Abdulhakem Y. Elezzabi \\ Editors
}

20-23 January 2008

San Jose, California, USA

Sponsored and Published by

SPIE

Volume 6892 
The papers included in this volume were part of the technical conference cited on the cover and title page. Papers were selected and subject to review by the editors and conference program committee. Some conference presentations may not be available for publication. The papers published in these proceedings reflect the work and thoughts of the authors and are published herein as submitted. The publisher is not responsible for the validity of the information or for any outcomes resulting from reliance thereon.

Please use the following format to cite material from this book:

Author(s), "Title of Paper," in Ultrafast Phenomena in Semiconductors and Nanostructure Materials XII, edited by Jin-Joo Song, Kong-Thon Tsen, Markus Betz, Abdulhakem Y. Elezzabi, Proceedings of SPIE Vol. 6892 (SPIE, Bellingham, WA, 2008) Article CID Number.

ISSN 0277-786X

ISBN 9780819470676

Published by

SPIE

P.O. Box 10, Bellingham, Washington 98227-0010 USA

Telephone +1 3606763290 (Pacific Time) · Fax +1 3606471445

SPIE.org

Copyright (c) 2008, Society of Photo-Optical Instrumentation Engineers

Copying of material in this book for internal or personal use, or for the internal or personal use of specific clients, beyond the fair use provisions granted by the U.S. Copyright Law is authorized by SPIE subject to payment of copying fees. The Transactional Reporting Service base fee for this volume is $\$ 18.00$ per article (or portion thereof), which should be paid directly to the Copyright Clearance Center (CCC), 222 Rosewood Drive, Danvers, MA 01923. Payment may also be made electronically through CCC Online at copyright.com. Other copying for republication, resale, advertising or promotion, or any form of systematic or multiple reproduction of any material in this book is prohibited except with permission in writing from the publisher. The CCC fee code is 0277-786X/08/\$18.00.

Printed in the United States of America.

Publication of record for individual papers is online in the SPIE Digital Library.

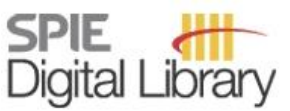

SPIEDigitalLibrary.org

Paper Numbering: Proceedings of SPIE follow an e-First publication model, with papers published first online and then in print and on CD-ROM. Papers are published as they are submitted and meet publication criteria. A unique, consistent, permanent citation identifier (CID) number is assigned to each article at the time of the first publication. Utilization of CIDs allows articles to be fully citable as soon they are published online, and connects the same identifier to all online, print, and electronic versions of the publication. SPIE uses a six-digit CID article numbering system in which:

- The first four digits correspond to the SPIE volume number.

- The last two digits indicate publication order within the volume using a Base 36 numbering system employing both numerals and letters. These two-number sets start with 00, 01, 02, 03, 04, 05, $06,07,08,09,0 \mathrm{~A}, 0 \mathrm{~B} \ldots \mathrm{OZ}$, followed by $10-1 \mathrm{Z}, 20-2 \mathrm{Z}$, etc.

The CID number appears on each page of the manuscript. The complete citation is used on the first page, and an abbreviated version on subsequent pages. Numbers in the index correspond to the last two digits of the six-digit CID number. 


\section{Contents}

vii Conference Committee

\section{SESSION 1 CARRIER AND EXCITON DYNAMICS I}

689205 Ultrafast piezospectroscopy in semiconductor nanostructures (Invited Paper) [6892-04] A. V. Akimov, A. V. Scherbakov, A.F. Ioffe Physico-Technical Institute (Russia);

T. Berstermann, Univ. of Dortmund (Germany); D. R. Yakovlev, A.F. Ioffe Physico-Technical Institute (Russia) and Univ. of Dortmund (Germany); P. J. S. van Capel, J. I. Dijkhuis, Utrecht Univ. (Netherlands); M. Bayer, Univ. of Dortmund (Germany)

\section{SESSION 2 PHONONS}

689206 Dynamics of LO phonons in InN studied by subpicosecond time-resolved Raman spectroscopy (Invited Paper) [6892-05]

K. T. Tsen, Arizona State Univ. (USA); J. G. Kiang, Uniformed Services Univ. of the Health Sciences (USA); D. K. Ferry, Arizona State Univ. (USA); H. LU, W. J. Schaff, Cornell Univ. (USA); H.-W. Lin, S. Gwo, National Tsing-Hua Univ. (Taiwan)

689207 Quantum confinement effects on the phonons of PbTe quantum dots in tellurite glasses [6892-06]

G. J. Jacob, D. B. Almeida, W. M. Faustino, E. Rodrigues, C. H. Brito, L. C. Barbosa,

C. L. Cesar, Univ. Estadual de Campinas (Brazil)

689208 Investigation of hot electrons generated from AIN/GaN-based high electron mobility transistor (Invited Paper) [6892-07]

S. K. Tripathy, G. XU, X. MU, Y. J. Ding, Lehigh Univ. (USA); K. Wang, Y. Cao, D. Jena, Univ. of Notre Dame (USA); J. B. Khurgin, Johns Hopkins Univ. (USA)

689209 Theory of electron-phonon interactions on nanoscales: semiconductor surfaces and two dimensional electron gases (Invited Paper) [6892-08]

N. Buecking, S. Butscher, M. Richter, Technische Univ. Berlin (Germany); C. Weber, Lund Univ. (Sweden); S. Declair, Technische Univ. Berlin (Germany); M. Woerner, K. Reimann, Max Born Institut für Nichtlineare Optik und Kurzzeitspektroskopie (Germany); P. Kratzer, Univ. Duisburg-Essen (Germany); M. Scheffler, Fritz-Haber-Institut der Max-Planck-Gesellschaft

(Germany); A. Knorr, Technische Univ. Berlin (Germany)

\section{SESSION $3 \quad$ NANOSTRUCTURES AND NANOPHOTONICS I}

6892 0C Ultra-fast dynamic control of the Q factor in a photonic crystal nanocavity (Invited Paper) [6892-11]

Y. Tanaka, J. Upham, T. Nagashima, T. Sugiya, T. Asano, S. Noda, Kyoto Univ. (Japan) 
6892 OD Resonant-tunnelling diodes beyond quasi-bound-state lifetime limit (Invited Paper) [6892-12]

M. N. Feiginov, D. Roy Chowdhury, Technische Univ. Darmstadt (Germany)

6892 OE Coherent acoustic phonons in nanostructures (Invited Paper) [6892-13]

T. Dekorsy, R. Taubert, F. Hudert, A. Bartels, A. Habenicht, F. Merkt, P. Leiderer, Univ. Konstanz (Germany); K. Köhler, J. Schmitz, J. Wagner, Fraunhofer Institute für Angewandte Festkörperphysik (Germany)

\section{SESSION 4 NANOSTRUCTURES AND NANOPHOTONICS II}

$6892 \mathrm{OH}$ Sub-picosecond polychromatic photoluminescence studies of CdSe and PbSe nanodots (Invited Paper) [6892-16]

C. Bonati, A. Cannizzo, F. van Mourik, M. Chergui, Ecole Polytechnique Fédérale de Lausanne, Lab. de Spectroscopie Ultrapide, ISIC (Switzerland)

\section{SESSION 5 NANOSTRUCTURES AND NANOPHOTONICS III}

6892 0J From cells to embryos: the application of femtosecond laser pulses for altering cellular material in complex biological systems (Invited Paper) [6892-18]

V. Kohli, A. Y. Elezzabi, Univ. of Alberta (Canada)

$6892 \mathrm{ON}$ Novel exact approach to polariton-polariton interactions and scattering rates: the limits of bosonization [6892-22]

M. A. Dupertuis, Ecole Polytechnique Fédérale de Lausanne (Switzerland); M. Combescot, Univ. Pierre et Marie Curie-Paris 6, CNRS (France); O. Betbeder-Matibet, Univ. Pierre et Marie Curie-Paris 6, Univ. Denis Diderot-Paris 7, CNRS (France)

\section{SESSION 6 SPINS AND SPINTRONICS}

6892 OP Femtosecond opto-magnetism (Invited Paper) [6892-24]

A. V. Kimel, A. Kirilyuk, T. Rasing, Radboud Univ. Nijmegen (Netherlands)

$68920 Q \quad$ Ultrafast photo-enhanced ferromagnetism in GaMnAs (Invited Paper) [6892-25]

J. Wang, I. Cotoros, Lawrence Berkeley National Lab. (USA) and Univ. of California, Berkeley (USA); X. Liu, J. K. Furdyna, Univ. of Notre Dame (USA); D. S. Chemla, Lawrence Berkeley National Lab. (USA) and Univ. of California, Berkeley (USA)

6892 OR Spinplasmonics: controlling plasmons at the quantum level (Invited Paper) [6892-26]

A. Y. Elezzabi, C. Baron, Univ. of Alberta (Canada); M. Johnson, Naval Research Lab. (USA)

\section{SESSION 7 SPECIAL TOPICS I}

$68920 \mathrm{U}$ Excitons and many-electron effects in the optical response of carbon nanotubes and other one-dimensional nanostructures (Invited Paper) [6892-29]

J. Deslippe, S. G. Louie, Univ. of California, Berkeley (USA) and Lawrence Berkeley National Lab. (USA) 


\section{SESSION $8 \quad$ NANOSTRUCTURES AND NANOPHOTONICS IV}

$68920 Z$ Ultrafast nonlinear spectroscopy of individual quantum dots: imaging and coherent coupling (Invited Paper) [6892-34]

J. Kasprzak, B. Patton, W. Langbein, Cardiff Univ. (United Kingdom)

\section{SESSION 9 SPECIAL TOPICS II}

689213 Kinetics of the Bose-Einstein condensation of microcavity polaritons (Invited Paper) [6892-38]

V. Savona, D. Sarchi, Ecole Polytechnique Fédérale de Lausanne (Switzerland)

\section{SESSION $10 \quad$ APPLICATIONS}

689216 Silicon-based nonlinear optical devices for high-speed optical communications (Invited Paper) [6892-39]

H. Rong, S. Ayotte, S. Xu, Intel Corp. (USA); O. Cohen, Intel Corp. (Israel); M. Paniccia, Intel Corp. (USA)

\section{SESSION $11 \quad$ WIDEGAP SEMICONDUCTORS}

689219 Four-wave mixing spectroscopy of ultraviolet excitons in strained GaN (Invited Paper) [6892-44]

T. Ishiguro, Y. Toda, S. Adachi, Hokkaido Univ. (Japan); S. F. Chichibu, Tohoku Univ. (Japan)

$68921 \mathrm{~A} \quad$ Ultrafast time-resolved photoluminescence of Zn-Se-Te multilayers with type-II ZnTe/ZnSe quantum dots [6892-45]

M. C-K. Cheung, I. R. Sellers, Univ. at Buffalo, SUNY (USA); I. L. Kuskovsky, Queens College at CUNY (USA); A. N. Cartwright, B. D. McCombe, Univ. at Buffalo, SUNY (USA)

\section{SESSION 12 THz SPECTROSCOPY AND APPLICATIONS I}

6892 1C Resonant-enhanced dipolar interaction between THz-photons and confined acoustic phonons in nanostructures (Invited Paper) [6892-47]

T.-M. Liu, J.-Y. Lu, C.-C. Kuo, M.-J. Yang, C.-W. Lai, P.-T. Chou, National Taiwan Univ. (Taiwan); M.-H. Chang, H.-L. Liu, National Taiwan Normal Univ. (Taiwan); Y.-T. Li, C.-L. Pan, National Chiao Tung Univ. (Taiwan); S.-H. Lin, C.-H. Kuan, C.-K. Sun, National Taiwan Univ. (Taiwan)

$68921 \mathrm{E}$ Terahertz-pulse emission through excitation of surface plasmons in metallic nanostructures (Invited Paper) [6892-49]

G. H. Welsh, K. Wynne, Univ. of Strathclyde (United Kingdom)

6892 IF Terahertz response of a two-dimensional electron gas (Invited Paper) [6892-50]

D. Golde, M. Kira, S. W. Koch, Philipps Univ. (Germany) 
$68921 G$ Designs of porous polymer THz fibers (Invited Paper) [6892-51]

A. Dupuis, A. Hassani, M. Skorobogatiy, École Polytechnique de Montréal (Canada)

$68921 \mathrm{H} \quad$ Ultrafast intraband relaxation in colloidal quantum dots (Invited Paper) [6892-53]

J. J. H. Pijpers, FOM Institute for Atomic and Molecular Physics (Netherlands); E. Hendry, Univ. of Exeter (United Kingdom); M. Bonn, FOM Institute for Atomic and Molecular Physics (Netherlands)

\section{SESSION 14 SURFACE AND INTERFACE}

$68921 \mathrm{~J}$ Carrier-envelope phase-controlled laser-surface interactions (Invited Paper) [6892-54]

P. Dombi, P. Rácz, Research Institute for Solid-State Physics and Optics (Hungary)

$68921 \mathrm{M}$ Electron relaxation dynamics at the In-rich (100) surface of InP [6892-57]

J. Szarko, L. Socaciu-Siebert, A. Neubaver, T. Hannappel, R. Eichberger, Hahn-MeitnerInstitut Berlin (Germany)

\section{SESSION 15 CARRIER AND EXCITON DYNAMICS II}

$68921 Q \quad$ Coherent optical processes of semiconductors studied via two-dimensional Fourier transform spectroscopy (Invited Paper) [6892-61]

A. D. Bristow, T. Zhang, S. T. Cundiff, Univ. of Colorado (USA) and National Institute of Standards and Technology (USA)

6892 is Direct observation of Landau damping with coherent plasmons in InSb [6892-63]

D. Seletskiy, M. P. Hasselbeck, M. Sheik-Bahae, L. R. Dawson, Univ. of New Mexico (USA)

POSTER SESSION

$68921 \mathrm{U}$ PbTe quantum dots grown by femtosecond laser ablation [6892-65]

E. Rodriguez, Univ. Estadual de Campinas (Brazil); D. Biggemann, Lab. Nacional de Luz Síncrotron, Lab. de Microscopia Eletronica (Brazil); L. Moya, W. A. Pippo, R. S. Moreira, D. Silva, C. L. Cesar, L. C. Barbosa, A. Schrank, C. R. Souza Filho, E. P. de Oliveira, Univ. Estadual de Campinas (Brazil)

Author Index 


\title{
Conference Committee
}

\author{
Symposium Chair
}

Ali Adibi, Georgia Institute of Technology (USA)

Symposium Cochair

James G. Grote, Air Force Research Laboratory (USA)

Program Track Chair

James G. Grote, Air Force Research Laboratory (USA)

Conference Chairs

Jin-Joo Song, University of California, San Diego (USA)

Kong-Thon Tsen, Arizona State University (USA)

Conference Cochairs

Markus Betz, Technische Universität München (Germany)

Abdulhakem Y. Elezzabi, University of Alberta (Canada)

Program Committee

Serge Bidnyk, Enablence, Inc. (Canada)

Majed Chergui, Ecole Polytechnique Fédérale de Lausanne (Switzerland)

David S. Citrin, Georgia Institute of Technology (USA)

Yujie J. Ding, Lehigh University (USA)

Arthur J. Fischer, Sandia National Laboratories (USA)

Jan A. Gaj, University of Warszawski (Poland)

Harald W. Giessen, Universität Stuttgart (Germany)

Costas P. Grigoropoulos, University of California, Berkeley (USA)

Robert A. Kaindl, Lawrence Berkeley National Laboratory (USA)

Dai-sik Kim, Seoul National University (South Korea)

Christoph Lienau, Carl von Ossietzky Universität Oldenburg (Germany)

Torsten Meier, Universität Paderborn (Germany)

Evgenii E. Narimanov, Purdue University (USA)

Janet L. Pan, Yale University (USA)

Chi-Kuang Sun, National Taiwan University (Taiwan)

Fabrice Vallee, Université Bordeaux I (France)

Chih-Chung Yang, National Taiwan University (Taiwan) 
1 Carrier and Exciton Dynamics I

Markus Betz, Technische Universität München (Germany)

Yujie J. Ding, Lehigh University (USA)

2 Phonons

Kong-Thon Tsen, Arizona State University (USA)

Thomas Dekorsy, Universität Konstanz (Germany)

3 Nanostructures and Nanophotonics I

Majed Chergui, École Polytechnique Fédérale de Lausanne (Switzerland)

Andreas Knorr, Technische Universität Berlin (Germany)

$4 \quad$ Nanostructures and Nanophotonics II

Martin Wegener, Forschungszentrum Karlsruhe (Germany)

Artur Zrenner, Universität Paderborn (Germany)

$5 \quad$ Nanostructures and Nanophotonics III

Fabrice Vallee, Université Claude Bernard Lyon 1 (France)

Jacek Kasprzak, Cardiff University (United Kingdom)

$6 \quad$ Spins and Spintronics

Stephan W. Koch, Philipps-Universität Marburg (Germany)

Martin Aeschlimann, Universität Kaiserslautern (Germany)

7 Special Topics I

Abdulhakem Y. Elezzabi, University of Alberta (Canada)

$8 \quad$ Nanostructures and Nanophotonics IV

Chi-Kuang Sun, National Taiwan University (Taiwan)

Steven G. Louie, University of California, Berkeley (USA)

9 Special Topics II

Jin-Joo Song, University of California, San Diego (USA)

Yujie Ding, Lehigh University (USA)

10 Applications

Donald J. Silversmith, Air Force Office of Scientific Research (USA)

11 Widegap Semiconductors

Chih-Chung Yang, National Taiwan University (Taiwan)

Kong-Thon Tsen, Arizona State University (USA) 
12 THz Spectroscopy and Applications I

Abdulhakem Y. Elezzabi, University of Alberta (Canada)

Mischa Bonn, FOM Institute for Atomic and Molecular Physics

(Netherlands)

$13 \mathrm{THz}$ Spectroscopy and Applications II

Markus Betz, Technische Universität München (Germany)

14 Surface and Interface

Rene Beigang, Fraunhofer-Institut für Physikalische Messtechnik (Germany)

15 Carrier and Exciton Dynamics II

Juergen Christen, Otto-von-Guericke-Universität Magdeburg (Germany)

Markus Betz, Technische Universität München (Germany) 
Downloaded From: https://www.spiedigitallibrary.org/conference-proceedings-of-spie on 26 Apr 2023

Terms of Use: https://www.spiedigitallibrary.org/terms-of-use 